\title{
Enseñanza del diseño mecánico mediante un proyecto CAD documentado
}

\section{Mechanical design teaching through documented CAD project}

\author{
ZAMARRIPA-MUÑOZ, Miguel Ángel†* \& OJEDA-ESCOTO, Pedro Agustín \\ Universidad Tecnológica de Aguascalientes \\ ID $1^{\mathrm{er}}$ Autor: Miguel Ángel, Zamarripa-Muñoz / ORC ID: 0000-0002-4589-1455, CVU CONACYT ID: 647582 \\ ID $1^{\text {er }}$ Coautor: Pedro Agustín, Ojeda-Escoto / ORC ID: 0000-0001-7282-4672, CVU CONACYT ID: 50027
}

DOI: $10.35429 / J M E .2019 .11 .3 .24 .32$

Recibido 28 Junio, 2019; Aceptado 30 Septiembre, 2019

\section{Resumen}

En el desarrollo de un proyecto del área metalmecánica existen dos vertientes de conocimientos, los teóricos, que se imparten de acuerdo a un plan de estudios en las universidades y por otro lado los prácticos, que normalmente se adquieren en el campo laboral. Para los ingenieros recién egresados, esto significa una desventaja al momento de competir por un puesto. Buscando lograr mejores resultados en el desempeño de los alumnos desde un plano real de diseño, se definió una estrategia para poder potenciar el aprendizaje de ambos conocimientos aplicándolos a un proyecto desarrollado de manera didáctica, cubriendo así temas que normalmente en un programa de estudios no se ven y generando toda la información necesaria para el diseño y fabricación del proyecto. En este artículo se reporta, como caso de estudio, el diseño de una grúa telescópica que integra las etapas desde la ingeniería conceptual hasta la ingeniería de detalle analizando toda la información que se genera entre éstas. Finalmente, se presentan los resultados preliminares de la información desarrollada para las diferentes etapas del proyecto ayudando así a los estudiantes en el proceso de toma de decisiones y organización de la información al desarrollar un proyecto de principio a fin.

Conocimientos teóricos, Conocimientos prácticos, proyecto

\begin{abstract}
In mechanical project development, there are two kind of knowledge, theoretical, taught according on syllabus at universities, and in the other hand, practical, usually learned in the working environment. For graduated recently engineers, it means a disadvantage once they are trying to get a job. Seeking to achieve better results on student performance from a real design plane, learning strategy is presented to enhance both knowledges applying them to an educational project completed, learning topics that normally is not covered on syllabus and generating all information required about design and manufacturing project. This paper shows a case of study about a telescopic crane design, analyzing all information generated from conceptual to detail engineering. Finally, preliminary results about all information generated through the different project stages is presented, helping in this way the decision-making process of the students and the information management when developing project from the beginning to the end.
\end{abstract}

Theoretical knowledge, Practical knowledge, project

Citación: ZAMARRIPA-MUÑOZ, Miguel Ángel \& OJEDA-ESCOTO, Pedro Agustín. Enseñanza del diseño mecánico mediante un proyecto CAD documentado. Revista de Ingeniería Mecánica. 2019. 3-11: 24-33

\footnotetext{
* Correspondencia del Autor ( miguel.zamarripa@utags.edu.mx)

$\dagger$ Investigador contribuyendo como primer Autor
} 


\section{Introducción}

Para desarrollar proyectos en el área metalmecánica se emplean una cantidad muy grande de variables que se tienen que controlar, organizar y documentar, la base fundamental de la resolución de problemas y realización del proyecto se puede resumir en los conocimientos teóricos y prácticos de los integrantes del equipo de trabajo. Los conocimientos teóricos, normalmente se aprenden de acuerdo a un plan de estudios en las universidades, y los prácticos normalmente se adquieren en el campo laboral. Para los estudiantes recién egresados esto significa un problema al momento de competir por un puesto, ya que la mayoría de las empresas solicitan experiencia ("conocimientos prácticos"). Sabiendo que la práctica no se puede separar de la teoría, se tiene que visualizar una solución global abarcando ambos temas y llevar a los estudiantes a un ambiente real de trabajo.

Con el objetivo de llevar al alumno a un plano real de diseño y realización de las actividades que se llevan a cabo en el desarrollo de un proyecto, se plantea el interactuar con la documentación realizada de un diseño de una grúa telescópica abordando temas desde la conceptualización del diseño, hasta los dibujos de fabricación de la misma, explicando así temas como: Organización de la información, filosofía de diseño, prácticas de manufactura, cálculos, materiales, técnicas de modelado 3D, dibujo de fabricación, consumo de materiales, etc.

En el ambiente laboral, normalmente la experiencia es lo que te ayuda a reducir tiempos en el desarrollo de un proyecto, por tal motivo, se plantea esta técnica enfocada a reducir tiempos de diseño y manufactura y ayudar así a las personas recién egresadas para que no les sea tan difícil colocarse en un puesto de trabajo.

Las etapas de desarrollo de un proyecto se pueden mencionar como: Definición de objetivos, ingeniería conceptual, ingeniería básica, ingeniería de detalle, manufactura y pruebas. En el apartado de "Procedimiento de diseño", se contempla ir analizando de manera cronológica los temas abordados en cada una de ellas. En un segundo apartado llamado "Caso de estudio" se analizan las diferentes etapas aplicadas al desarrollo del diseño de la grúa telescópica.

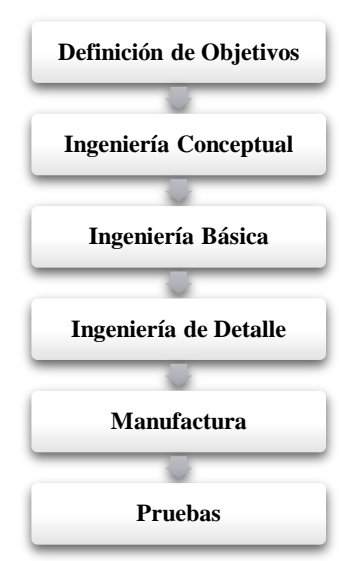

Figura 1 Desarrollo de proyecto

Fuente: Elaboración Propia

\section{Objetivos}

El propósito principal de la investigación radica en incrementar los conocimientos prácticos del estudiante mediante el desarrollo de un proyecto real y de magnitudes poco alcanzables en los planes de estudios debido al tiempo, ayudándolo a interactuar con grandes contenidos de información y saber documentarlo.

\section{Metodología}

Uno de los grandes problemas a la hora de diseñar, es el fundamentar los diseños en base a la manufactura, es muy recurrente el diseñar sin considerar el ¿cómo lo voy a fabricar?, esto ocasiona muchas pérdidas de tiempo trabajando las piezas más de una vez para corregir los errores o incluso realizar un rediseño de componentes y maquinar nuevas piezas. Bajo esta condición, se considera el uso de un proceso denominado como "Diseño para X", el cual reúne una serie de técnicas de diseño que se introducen en la fase preliminar con el objeto de que el producto final cumpla una serie de características deseables durante el ciclo de vida del desarrollo del producto para garantizar su calidad. Dentro de las técnicas que comprende este proceso, se puede identificar la de "Diseño para manufactura" (DFM), el cual se emplea como línea principal en este proceso de enseñanza.

Para el desarrollo de toda esta información se utilizó como base principal el software de diseño Solidworks desarrollado por Dassault Systemes. 


\section{Procedimiento del diseño}

\section{Definición de objetivos}

La claridad y precisión en la definición de estos objetivos son fundamentales en el desarrollo del proyecto, ya que de esto depende la evaluación de los resultados que se obtengan al final. Cabe mencionar que en el desarrollo del proyecto se está expuesto a perder el sentido de los objetivos planteados desde el inicio, por lo que se debe tener un constante monitoreo y no perder la focalización de éstos.

\section{Ingeniería conceptual}

Generalmente un equipo de diseño está integrado por personal de varias disciplinas, esta situación ayuda a ver el problema desde varias perspectivas y a la vez se pueden ver diferentes escenarios para realizar el diseño conceptual. Para la realización de esta etapa se considera abordar temas teóricos como: Procesos de manufactura disponibles, estudio del arte, lluvia de ideas, seguridad y filosofía de diseño. Estos temas nos apoyan para marcar el camino en la toma de decisiones.

Por la parte práctica, se requiere considerar los temas referentes a la viabilidad del diseño, generalmente las personas con más experiencia en el equipo de trabajo son los que tienen un panorama más amplio para acotar estos criterios, mencionándolos por prioridad tenemos lo siguiente:

1. Funcionalidad: Es el tema primordial, ya que de aquí parte el objetivo planteado en la primera etapa del proyecto y define la necesidad que se tiene que resolver.

2. Costo de manufactura: Es fundamental el considerar como se va a fabricar, para solventar la necesidad con el presupuesto asignado.

3. Costo de materiales: Es crítico el análisis de los materiales, ya que están ligados con el tema de disponibilidad del material y costos.

4. Exceso de dimensiones: El realizar un diseño modular es fundamental para no caer en problemas de movilidad.

5. Estética: Por último y como tema también relevante es un producto que tiene que ser atractivo al cliente para poder comercializarlo.
A manera complementaria de estos temas, y considerando la esencia de esta etapa del proyecto que es conceptual, se consideran los temas de: manejo de la información que nos proporciona un orden y rastreabilidad de la información y el modelado rápido en 3D que nos permite analizar diferentes soluciones de manera conceptual.

\section{Ingeniería básica}

Esta ingeniería es una profundización del análisis realizado en la ingeniería conceptual previa, cuyo resultado son los datos de entrada para esta etapa del diseño. En el desarrollo de la ingeniería básica se analizan los siguientes temas considerados como conocimientos teóricos: trigonometría, análisis de fuerzas, momentos, elemento finito, resistencia de materiales, modelado 3D, hidráulica, neumática, selección de equipo comercial, etc.

Siendo esta una de las etapas más nutridas en cuanto a conocimiento práctico para aplicar, ya que la experiencia es la que abre la perspectiva para la toma de decisiones al momento de estar diseñando, los temas abordados en esta etapa son:

Materiales: Selección de materiales comerciales existentes y con disponibilidad.

Prácticas en tecnología láser: Consideraciones para el corte laser de placas aprovechando todas las ventajas que nos proporciona este proceso de manufactura tales como: uso de ranuras y protuberancias en piezas para su armado, etc.

Prácticas de doblez: Consideraciones para el doblez de chapa metálica, entre las que se pueden mencionar: relación entre espesores y radios de doblez, configuraciones de dados, etc. Esto con el objetivo de reducir tiempos de diseño y manufactura.

Transporte: Validación de las dimensiones generales de lo desarrollado para garantizar su movilidad sin problemas, y de ser necesario plantear un diseño modular.

Manejo de la información: Nomenclatura de componentes utilizados en el proyecto, organización de carpetas para la clasificación de la información, respaldos de información, etc. 


\section{Ingeniería de detalle}

Es la etapa en la cual se analizan todos y cada uno de los componentes del diseño minuciosamente mediante el dibujo de fabricación, el análisis de los temas para esta etapa se pueden abordar como:

\section{Teóricos:}

1.1) Tolerancias dimensionales y geométricas: Empleo de tablas de ajustes recomendados ISO, y análisis de las tolerancias geométricas requeridas para el diseño, garantizando su correcto ensamble.

1.2) Acotación funcional: Análisis de acotación bajo un concepto funcional de las piezas y ensambles para reducir costos de manufactura.

1.3) Dibujo de mecánico: Normatividad de dibujo (vistas, cortes, secciones, escalas, revisiones, listas de partes, etc.). Dibujo de fabricación de componentes y ensambles.

\section{Prácticos:}

2.1) Técnicas para reutilización de dibujos y modelos 3D: El empleo de un software de diseño tiene muchas ventajas y un factor principal es tener bien claro la forma como funciona y las ventajas que nos brinda para la reutilización de dibujos y modelos 3D para ahorrar tiempo de diseño.

\section{Manufactura}

En la etapa de ingeniería conceptual se definieron los procesos de manufactura disponibles para el proyecto, por lo que esta etapa estará totalmente ligada a lo que se planteó de forma conceptual.

En cuanto a los conocimientos teóricos para esta etapa se abordan temas de soldadura, tratamientos superficiales, metrología y procesos de mecanizado.

Por la parte práctica se pretende concientizar al alumno de los procesos de manufactura más habituales tales como el uso de las máquinas-herramientas y abordar temas como:
1) Clasificación de los materiales para el procesado: Dentro del taller se debe de tener bien identificado el flujo de los materiales y en qué orden procesarlos, para poder aprovechar todos los recursos en el menor tiempo posible.

2) Consumo de materiales: Proveer al alumno de técnicas para identificar rápidamente el consumo de material de fabricación que se va a consumir en el proyecto, ya que en ocasiones este análisis toma mucho tiempo para realizar las compras de lo que se requiere para el proyecto.

\section{Pruebas}

Esta etapa se centra en la validación de los resultados del diseño realizado, evaluando así, que lo desarrollado cumpla con los objetivos definidos en la etapa 1. Para ello se tiene que ser muy puntual en la evaluación de los resultados y validar el correcto funcionamiento de lo diseñado. Como parte del conocimiento práctico de esta etapa y a su vez fundamental para el equipo de trabajo es la aplicación de una práctica conocida como "lecciones aprendidas", en la que se les da respuesta a las siguientes preguntas:

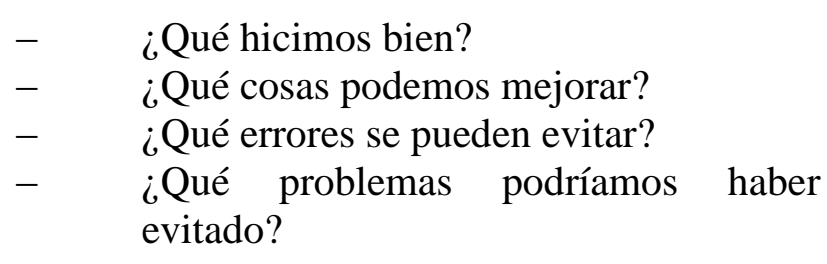

Documentando estas acciones se tiene como resultado:

No cometer los mismos errores.
$-\quad$ No "reinventas la rueda" en cada
proyecto nuevo.
Repites los éxitos.

Y todo esto se traduce a un aprendizaje del equipo. Siendo esto un enriquecimiento fundamental en cada integrante del equipo.

\section{Caso de estudio}

Una vez analizados los temas que se emplean en cada una de las etapas de diseño de un proyecto, toca el turno de estudiar las diferentes etapas aplicadas a un proyecto documentado, para ello se plantea el diseño de una grúa telescópica con el fin de ejemplificar de una manera puntual las consideraciones antes mencionadas. 


\section{Definición de objetivos}

Para el caso de estudio se define como objetivo: Realizar el diseño de una grúa telescópica con capacidad de carga de $3,000 \mathrm{~kg}$ y un alcance de vertical de izaje de $6 \mathrm{~m}$.

\section{Ingeniería conceptual}

Definición de procesos de manufactura disponibles: Para la realización de la grúa se definen los siguientes procesos de manufactura:

$\begin{array}{ll}- & \text { Corte segueta cinta } \\ - & \text { Torneado } \\ - & \text { Taladrado } \\ - & \text { Fresado } \\ - & \text { Corte laser } \\ - & \text { Doblez de chapa } \\ - & \text { Soldadura } \\ - & \text { Pintura }\end{array}$

Mediante una lluvia de ideas (brainstorm) se exponen los factores a considerar para el diseño, exponiendo temas como:

Estudio del arte, que consiste en la revisión de los modelos existentes de grúas en el mercado y analizar las configuraciones existentes tomando en cuenta sus ventajas y desventajas.

En la filosofía de diseño se analizan los 10 aspectos vulnerables particularmente para cada proyecto que no pueden ser ignorados sin riesgo de fracaso:

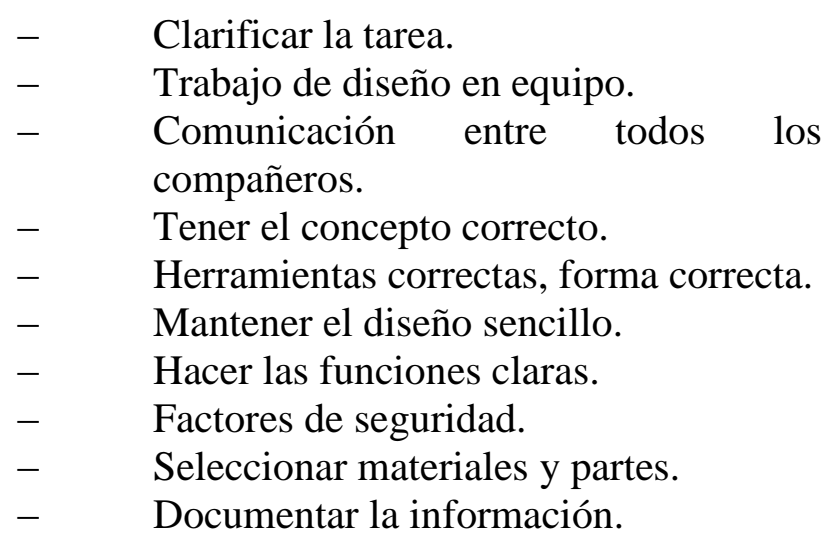

Una vez analizados estos temas se procede a modelar en 3D un diseño conceptual rápido, el cual ayudará a comenzar el análisis de alcances y longitudes de los componentes de la grúa.
Dicho modelo, debe garantizar la funcionalidad de la grúa, que los componentes sean factibles de fabricarlos bajo los procesos de manufactura mencionados anteriormente, verificar que los componentes sean de materiales comerciales (no fabricación especial) y con disponibilidad, que el ensamble(s) no exceda dimensiones de transporte para garantizar su movilidad y que sea atractivo visualmente para comercializarlo.

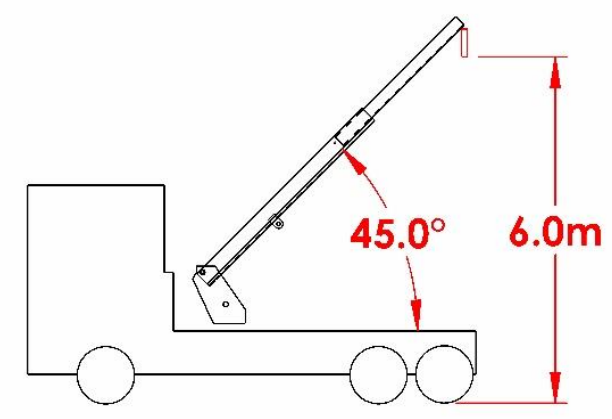

Figura 2 Ingeniería conceptual

Fuente: Elaboración Propia

\section{Ingeniería básica}

Después de analizar los alcances de la grúa en la etapa anterior, se procede a identificar la configuración del brazo y la selección de materiales para la construcción del mismo. El brazo se diseñará bajo las consideraciones siguientes:

Se considera el uso de material acero estructural rectangular (PTR) para la extensión de la grúa tratando de reducir costos de manufactura.

El uso de placas dobladas para la construcción del brazo principal ayudará a controlar geometría, tolerancias, distribución de esfuerzos y sujeciones de los componentes.

- La altura promedio de la plataforma de un camión se considera de 50 pulgadas con respecto al nivel del piso. 
- Realizar el diseño 3D de forma simplificada con el objetivo de realizar un análisis de elemento finito considerando las dimensiones y cargas definidas en las etapas anteriores obteniendo así la geometría que nos proporcionará la resistencia necesaria del brazo, considerando un factor de seguridad de 3 aplicado como una carga repetida en una dirección basado en el límite de fluencia del material [2], y el uso de un material común como el ASTM A36 con un límite de fluencia de 36,300 psi [12], y de esta forma se realiza la evaluación del diseño (ver figura 3).

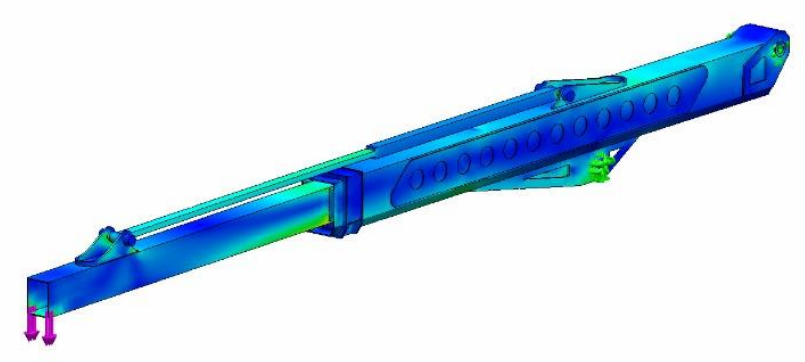

Figura 3 Análisis de elemento finito Fuente: Elaboración Propia

Una vez encontrando los valores óptimos de esfuerzos y reforzando las partes críticas en el diseño, se procede a obtener el peso total del brazo, el cual nos servirá para el cálculo del cilindro de levante [1], que es el componente medular del funcionamiento de la grúa.

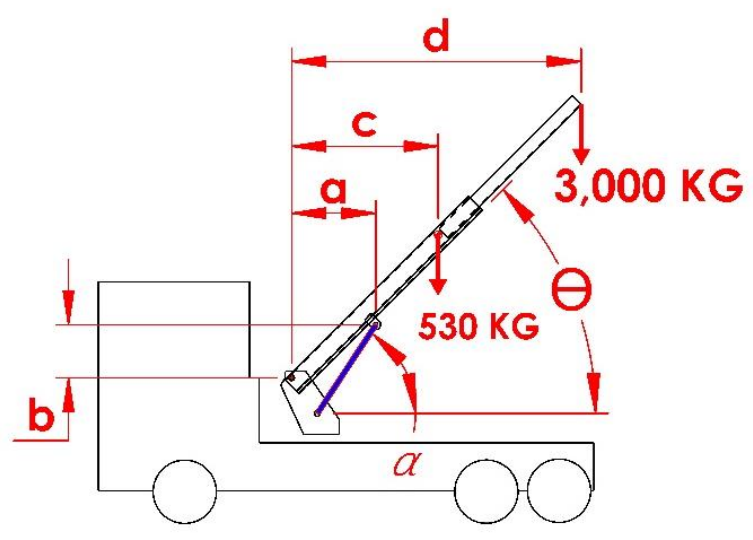

Figura 4 Cálculo cilindro de levante Fuente: Elaboración Propia

Considerando un peso aproximado del brazo de $530 \mathrm{~kg}$ y considerando que el ángulo más crítico para la fuerza del brazo es $\theta=0^{\circ}$ (ver figura 4), se tiene lo siguiente:
$\sum_{\mathrm{MA}}=0=(\mathrm{a})\left(\mathrm{F}_{\mathrm{CIL}} \operatorname{Sen} \alpha\right)+$

(b) $\left(\mathrm{F}_{\mathrm{CIL}} \operatorname{Cos} \alpha\right)-(\mathrm{c})(530 \mathrm{~kg})-$

$(d)(3000 \mathrm{~kg})=0$

$\mathrm{F}_{\mathrm{CIL}}=20573.6 \mathrm{~kg}=45261.9 \mathrm{lbs}$

Encontrando el diámetro del cilindro tenemos:

$P=\frac{\mathrm{F}}{\mathrm{A}}=\frac{4 \mathrm{~F}}{\pi d^{2}}$

Considerando que el sistema hidráulico funcione a una presión de 2450 psi tenemos:

$$
\begin{aligned}
& d=\sqrt{\frac{4 F}{\pi P}}=\sqrt{\frac{4(45261.9 l b s)}{\pi\left(2450 l b s / p l g^{2}\right)}} \\
& d=4.84 \mathrm{plg} \approx 5 \mathrm{plg}
\end{aligned}
$$

Una vez evaluado el brazo bajo los criterios mencionados en el análisis de elemento finito y el cálculo del cilindro, se realizan las consideraciones para la manufactura:

Materiales: Se requiere tener conocimiento de las medidas de materiales comerciales con disponibilidad, ya que a menudo encontramos en catálogos de proveedores materiales que no son comunes de comercializar y tienen tiempos de entrega largos.

Corte laser: Este proceso está acotado al corte de piezas que se obtienen a partir de hojas de lámina o placa con un espesor preferentemente de hasta $5 / 8$ pulgadas. En este proceso se tiene que hacer una serie de consideraciones, ya que se pueden obtener muchas ventajas para el ensamble de los componentes haciendo uso de ranuras y protuberancias en las piezas para facilitar los escuadres de las piezas, sobretodo en ensambles de soldadura, evitando así el uso de escantillones.

Otra consideración importante es acerca del barrenado de las piezas, no se puede cortar un barreno más pequeño que el espesor de la placa, estas prácticas nos ayudarán a reducir tiempos de manufactura en gran medida. 
Doblez: Para facilitar el proceso de doblez, se puede hacer uso una ventaja muy importante que nos proporciona el corte laser, ya que el diseñador puede colocar marcas en el material en los lugares donde va a doblarse, ayudando así a reducir tiempos en el proceso de doblez convencional, ya que en muchas ocasiones el operador de la dobladora tiene que marcar la pieza para posteriormente colocar la pieza para doblarla.

Transporte: En esta etapa se procede a la validación del dimensionamiento de los ensambles diseñados para evitar tener problemas de movilidad.

Manejo de la información: Es de vital importancia la clasificación de la información generada. De forma general para un proyecto podemos realizar la siguiente clasificación:

$\begin{array}{ll}- & \text { Administración } \\ - & \text { Respaldos } \\ - & \text { Cálculos } \\ - & \text { Catálogos } \\ - & \text { Información de control (electrónica) } \\ - & \text { Datos de entrada } \\ - & \text { Modelos 3D } \\ - & \text { Imágenes y videos }\end{array}$

Es de gran importancia generar una nomenclatura adecuada a los documentos generados, modelos 3D (tornillería, partes comerciales, elementos para manufactura, etc.), catálogos, respaldos, órdenes de compra, etc. con la finalidad de tener un control de manufactura y a su vez la rastreabilidad de la información.

\section{Ingeniería de detalle}

En esta etapa se realiza el dibujo de fabricación de la grúa, desnudando así el diseño a su máxima expresión, ya que de los temas principales para esta etapa son las tolerancias asignadas para el correcto ensamble de los componentes teniendo bien claro que el acotado funcional es el que nos provee el mayor beneficio, ya que nos permite reducir costos de fabricación haciendo énfasis en los puntos críticos para el ensamble. Es fundamental el dominio del dibujo mecánico como lenguaje universal, ya que el objetivo del dibujo es documentar toda la ingeniería desarrollada.
En cuanto a los conocimientos prácticos aplicados en esta etapa, se debe tocar el tema de tener buen dominio del software utilizado para realizar el diseño y saber el vínculo que existe entre los dibujos generados y los modelos 3D, ya que esto nos permite manipular la información de tal forma que se puede reutilizar la información generada para crear nuevos componentes con cierta similitud tales como pernos, bujes, etc. Y extrapolando éste criterio a cualquier ensamble o pieza.

Normalmente un alumno durante su carrera no le es posible aplicar todas las consideraciones necesarias en el diseño de un proyecto de esta magnitud, bajo esta iniciativa se pretende aplicar los conocimientos en un proyecto ya desarrollado como lo es el diseño de la grúa telescópica.

\section{Manufactura}

Como anteriormente se mencionó, el factor crítico es el flujo de material en el taller de manufactura ya que se pueden realizar procesos en paralelo y que al final converjan en el ensamble de los mismos. Para la manufactura de la grúa, hay dos vertientes de tipos de material, los que se obtienen a partir de placas (hojas de lámina) y los materiales que se compran por longitud (acero estructural, material redondo, tubo, etc.) y los dos procesos fundamentales al inicio de la fabricación son: corte laser (para el procesado de la placa) y el proceso de corte por segueta (para el procesado del material como acero estructural, etc.), de ésta forma se puede comenzar la clasificación del material para procesarlo en paralelo, ya que los procesos no dependen uno del otro.

Estos dos procesos son el punto de partida de la materia prima, y a su vez, están surtiendo de material a los demás procesos como doblez, torneado, fresado, soldadura, etc. Por otro lado, es de vital importancia el enfatizar en concientizar al alumno acerca del uso de herramientas para identificar el consumo de material, bajo las consideraciones que se está trabajando con placas surtidas por pieza (largo por ancho), se requiere definir la cantidad de placas que se requiere comprar. Para identificar el consumo de placas se procede a realizar un anidado (manual o automático en software especializado) realizando el acomodo óptimo de las piezas dentro de la placa comercial (ver figura 5). 
El punto de partida para este proceso es la generación de archivos “.dxf” (archivo 2D) de las piezas que van a fabricarse a partir de placas, posteriormente se utilizarán estos archivos para su anidado correspondiente.

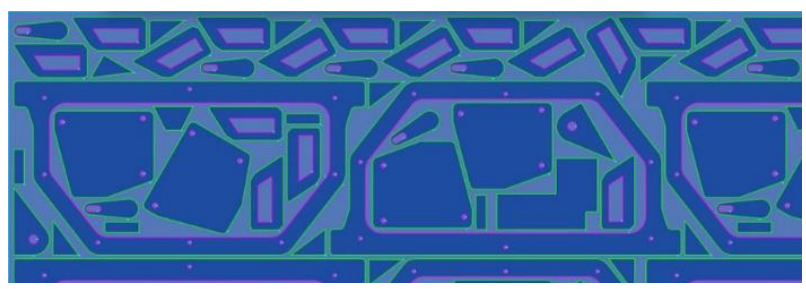

Figura 5 Anidado de chapa metálica. [13]Sigmanest.

Por otro lado, se encuentran los materiales que se obtienen a partir de tramos de material, dentro de los que podemos mencionar son acero estructural como PTR, ángulos, vigas, redondos, etc. Para calcular el consumo de materiales de este tipo, existen softwares que calculan el consumo óptimo, para que no haya desperdicios considerables, sobre todo cuando se trata de un producto fabricado en serie (ver figura 6).

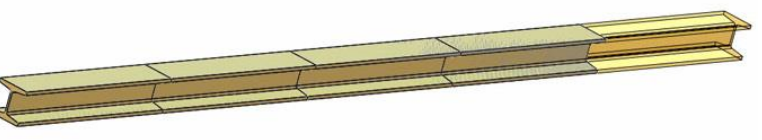

Figura 6 Anidado de cortes por longitud. [13]Sigmanest.

\section{Pruebas}

Como se ha mencionado anteriormente, el objetivo de esta metodología es involucrar al alumno con un diseño real y llevarlo a considerar todos los factores al diseñar un proyecto y como resultado del mismo se muestra el diseño de la grúa desarrollada como un proyecto de análisis para los alumnos (ver figura 7).

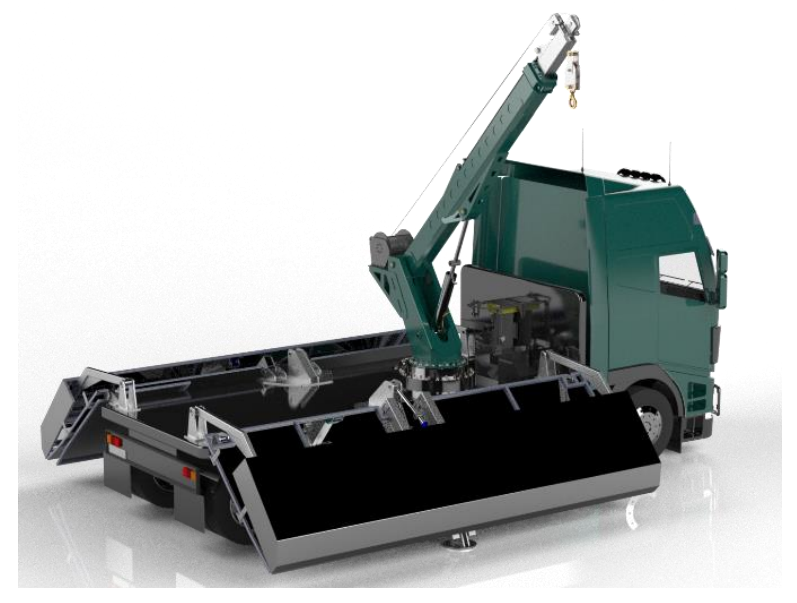

Figura 7 Diseño final de prototipo Fuente: Elaboración Propia

\section{Resultados}

Como resultado de la información generada, se presenta el diseño preliminar de la grúa desarrollada para que funcione como banco de análisis para los alumnos, y de esta forma fortalezcan sus conocimientos y les ayude para la solución de problemas y organización de la información generada en el proyecto, planteándose la información de manera genérica para aplicarla a cualquier proyecto del área metal-mecánica.

Es de vital importancia proveer a los alumnos estrategias para desarrollar sus ideas y documentarlas de forma correcta, incentivando la innovación y fortaleciendo las oportunidades de los estudiantes.

A continuación, se describe una breve reseña de los resultados obtenidos para cada una de las etapas del estudio:

1. Definición de objetivos: Planteamiento de los diferentes escenarios o perspectivas que se pueden ver al iniciar un proyecto.

2. Ingeniería conceptual: Involucrar al alumno con los conceptos importantes en la toma de decisiones para trazar el rumbo del proyecto.

3. Ingeniería básica: En esta etapa se tiene una gran influencia en los conocimientos prácticos y es donde se obtienen los mayores beneficios de esta metodología, ya que se abordan consideraciones de manufactura que ayudarán en gran medida al proceso de fabricación y tiempos del proyecto.

4. Ingeniería de detalle: Es de gran importancia el saber documentar de forma detallada toda la información generada en un proyecto ya que esto es lo que le da valor al diseño de ingeniería, la información tiene que ser controlada y resguardada de forma correcta para poder replicar o reutilizar esta información posteriormente, ya sea para mejorar el diseño, o bien, para utilizarla en otro proyecto posterior. El alumno deberá comprender la importancia de esta etapa.

5. Manufactura: Cuando sabemos con cuantas máquinas contamos para realizar el trabajo, es vital saber darle el flujo al material de forma correcta para que no se produzcan cuellos de botella y de esta forma agilizar el proceso de fabricación. 
6. Pruebas: Sabiendo de antemano que el proyecto está enfocado a la docencia, esta etapa se tiene que llevar de manera teórica esperando contar posteriormente con recurso para la validación del diseño.

\section{Contribución de la investigación}

En el proceso de aprendizaje de los alumnos en las universidades, normalmente se interactúa con proyectos pequeños que pueden desarrollar durante una asignatura, pero pocas veces interactúan con un proyecto de mayor magnitud como el que se plantea en este artículo. Este método de enseñanza contribuye a ampliar el panorama del estudiante acerca de todas las variables que debe de considerar al realizar un diseño en sus diferentes etapas de una manera más amplia, clara y palpable.

\section{Agradecimiento}

Los autores del presente artículo agradecen el apoyo brindado por la Universidad Tecnológica de Aguascalientes para realizar este estudio.

\section{Conclusiones}

Generalmente los estudiantes recién egresados buscan oportunidades en el campo laboral para aprender los conocimientos que nos da la práctica (experiencia), la metodología descrita en este artículo está enfocada para poder brindar al alumno fortalezas para la toma de decisiones en el desarrollo de un proyecto abordando tanto los conocimientos teóricos como prácticos.

Un factor fundamental en un equipo de diseño es el trabajo y la interacción del equipo para obtener los resultados esperados, cada uno de los integrantes del equipo debe mantener su mente abierta y libre de prejuicios, ya que la generación de ideas es fundamental para encontrar la solución correcta al reto de cada proyecto.

El conocimiento adquirido por los estudiantes bajo esta metodología, les ha servido a visualizar el diseño desde una perspectiva de manufactura, considerando los procesos de fabricación definidos desde el inicio del proyecto y proponer soluciones viables y reales. Sin duda, esto es una aportación a sus conocimientos prácticos para enfrentarse a la vida laboral.

\section{Referencias}

[1] Beer F., Johnston R. \& Eisenberg E. (2007). Mecánica Vectorial para ingenieros. Estática. México D.F.: McGraw-Hill.

[2] Faires V. (1997). Diseño de elementos de máquinas. México D.F.: Limusa.

[3] Pugh, S. (1990). Total Design. Great Britain: Addison Wesley.

[4] Ullman, D. G. (1992). The Mechanical Design Process. New York: Mc. GrawHill.

[5] Ulrich K. T. \& Eppinger S.D. (1995). Product design and development. International Editions: Mc. Graw-Hill.

[6] Hubka, V. \& Eder, W.E. (1992). Engineering Design - General Procedural Model of Engineering Design. Zürich: Edition Heurista.

[7] Pahl, G. \& Beitz, W. (1996). Engineering Design - A Systematic Approach. Great Britain: Springer-Verlag.

[8] Evbuomwan, N.F.O., Sivaloganathan, S. \& Jebb, A. (1996). A Survey of Design Philosophies, Models, Methods and Systems. Proceedings of the Institution of Mechanical Engineers, Part B: Journal of Engineering Manufacture, 210, 301-320.

[9] Ullah, I., Tang, D. \& Yin, L. (2016). A literature overview, 9th International Conference on Digital Enterprise Technology - DET 2016 - "Intelligent Manufacturing in the Knowledge Economy Era", Procedia CIRP, 56, 25-33.

[10] Finger, S. \& Dixon, J. (1989). A Review of Research in Mechanical Engineering Design. Part I: Descriptive, Prescriptive, and Computer-Based Models of Design Processes, Research in Engineering Design, Springer-Verlag New York Inc., 1, 51-17. 
[11] Finger, S. \& Dixon, J. (1989). A Review of Research in Mechanical Engineering Design. Part II: Representations, Analysis, and Design for the Life Cycle, Research in Engineering Design, Springer-Verlag New York Inc., 1, 121-137.

[12] http://www.matweb.com

[13] https://www.sigmanest.com/features/ 